\title{
TEMPERATURE GRADIENT ANALYSIS BY THERMOGRAPHY USED IN OPTOMETRY
}

\author{
Dana Rizescu ${ }^{1}$, Ciprian Ion Rizescu ${ }^{2}$ \\ 1,2University POLITEHNICA of Bucharest, Mechatronics and Precision Mechanics Department \\ dana.rizescu@upb.ro
}

\begin{abstract}
This paper is a highlight of the results regarding the ocular temperature held so far by the scientific community. The authors developed an experimental study on a number of 20 subjects. Infrared (IR) ocular thermography determines ocular surface temperature (OST) of the eye and preorbital skin by measuring the amount of IR radiation emitted from the surface with an infrared thermal imaging camera. Noninvasive ocular thermography was first introduced in 1968 and was used to evaluate both normal and pathological conditions. For this, a FLIR Systems camera was used to capture the eye temperatures of the 20 young subjects (male, female). IR-ray thermography is recognized, being a method of analyzing the temperature of the non-invasive eyeball. Following the analysis of the experimental data, the results obtained by other previous studies were confirmed. The acquisition of corneal center temperatures (manually determined) in both eyes led to an average temperature of $34.5^{\circ} \mathrm{C}$, an inter ocular difference of $0.110{ }^{\circ} \mathrm{C}$. Also, a maximum temperature was found in the tear duct, relative to the center of the cornea and the lateral corner. The Flir camera could also be used in the study of contactology, of the efficient adaptation of the patient with the different types of contact lenses.
\end{abstract}

Keywords: Ocular Surface Temperature, Thermography, Infrared Detection.

\section{Introduction}

Thermography is a non-invasive method of taking the surface temperature of the eye by acquiring infrared images, which is why it can also be used by the optometrist. Beginning with Mapstone, 1960, this method has also been used in ophthalmology. Noninvasive ocular thermography was used to evaluate both normal and pathological conditions

The ocular thermography has undergone a remarkable evolution in the last decades, both due to the emergence of the new means of biomedical investigation accessible at a lower price, as well as due to the increasing desire to offer high comfort to the patient. For this, IR-ray thermography is recognized, being a method of analyzing the temperature of the non-invasive eyeball. So far, thermography has been used in the study of the human system of tear drainage, dry eye syndrome, carotid artery stenosis, glaucoma, unilateral exophthama, and more.

For example, according to a study published by F. Galassi, glaucoma patients were found to have a lower temperature than healthy subjects, with the temperature being measured at 5 points on the central corneal line. [1], [2].
Another important aspect of the study of eye temperature is the connection that could be established between the determined temperature and the thickness of the lipid layer of the tear film, a study by Giraldez M. showing that a high value of the corneal temperature indicates a thicker layer. [3]

External factors that explain the appearance of the temperature differences:

The interocular difference.

- It is certified that there is an eye disease if there is a difference in temperature between 2 eyes greater than $0.6^{\circ} \mathrm{C}$. [2]

- Difference in nasal / temporal temperature.

The temperature of nasal conjugation is higher than temporal due to richer vasculature.

- Age, sex, Eye color.

It is considered a decrease of approximately $0.01{ }^{\circ} \mathrm{C}$ / year. Brown eyes have, generally, a temperature below the blue eyes. Sex does not influence.

Internal factors that explain the appearance of the temperature differences:

- Elements of pathology (glaucoma)

One can observe that there is a decrease in temperature values for glaucoma patients. Also, ischemia / hyperemia of one of the eyes may induce a significant ocular difference. 
- Tear film thickness

There is known that the dependence of the thickness of the lipid layer in the tear film on the value of the ocular surface temperature (OST) is supported. An increased value of OST in normal subjects is associated with greater thickness of this layer [3], [4].

\section{Experimental Setup}

In this study, a Flir Systems camera with a FPA (focal plane array) detector, a resolution of $120 \times 120$ pixels and an accuracy of $\pm 0.2^{\circ} \mathrm{C}$. The camera is presented in figure 1.

\section{Ambient temperature}

Prior to the measurement, each patient had an accommodation time with the laboratory temperature of about 10 minutes. The ambient temperature had values ranging from 21.3 to $23.4^{\circ} \mathrm{C}$.

\section{Measuring distance}

For the accuracy of the results, measurements were made by one single subject at a distance of about 200/300 mm.

Measuring time

There was developed a study concerning temperature variation after a certain time range. There were measured the temperatures after $1,3,6$, 9 [s] from the last blinking.

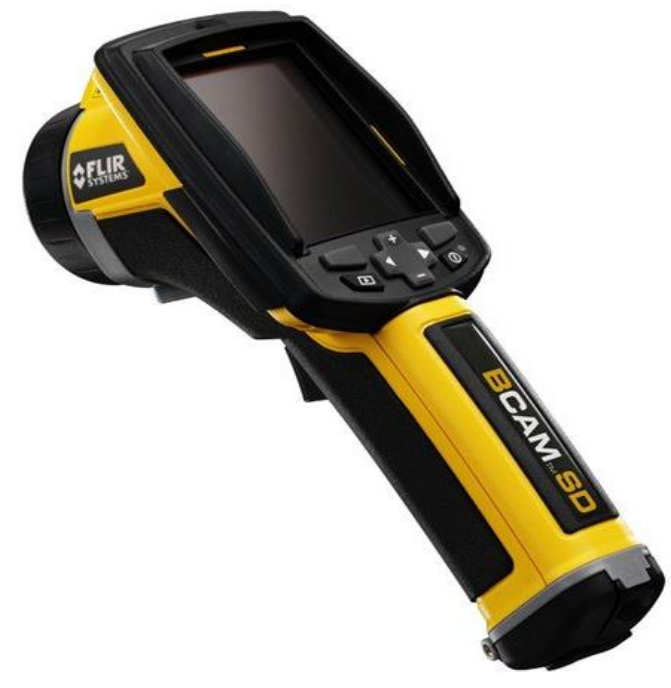

Figure 1: FLIR Camera

Measurements developed with FLIR camera:

- Measurements with eyes closed - in center;

- Measurements in the center of the cornea in the interval between flashes of 1-3-6-9 seconds;

- Measurements at the caruncula lacrimalis;

- Measurements at the level of the palpebral fissure.

\section{Experimental Results}

Before each examination, room temperature, humidity and air flow were recorded, to make sure to have relatively constant environmental parameters. Also, at each examination, the subject was requested to keep the eyes closed for 3-5 s, then to open both eyes wide.

In the subsequent figures 2-5 there are presented some results, obtain with FLIR camera as it is shown:

- Temperature in the geometric center of the cornea, figure 2 .

The surface temperature of the cornea is determined by adjacent ocular and extra-ocular tissues- which is an internal environment - and by conditions prevailing external to the body-an external environment. Any investigation of their effects is rendered more complex than would otherwise be the case in that the external environment is intermittently excluded during blinking so that, when the lids are closed, the thermal environment of the cornea is exclusively internal [5],[6].

Other important results are presented in figures 3,4 and 5 .

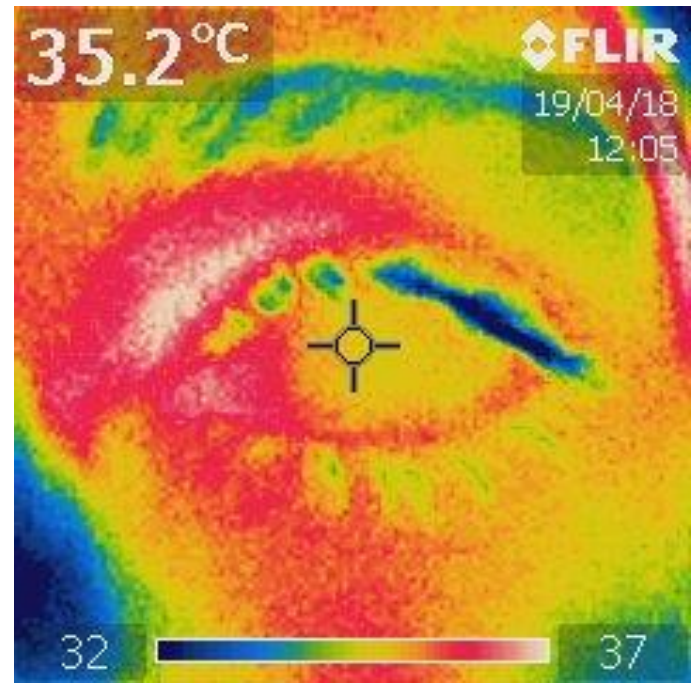

Figure 2: Temperature in the geometric center of the cornea

- Temperature of the palpebral fissure, figure 3.

- Temperature for closed eyelids, in front of center cornea, figure 4.

- Other important temperature is the caruncula lacrimalis one as it is shown in figure 5 .

There were considered both male and female subjects, with age from 20 to 25 years old. The measurements were achieved in the laboratory with ambient temperature in the range $20-22^{\circ} \mathrm{C}$.

There were determined temperature variation for male/female subjects: centre / caruncula lacrimalis and palpebral fissure. 


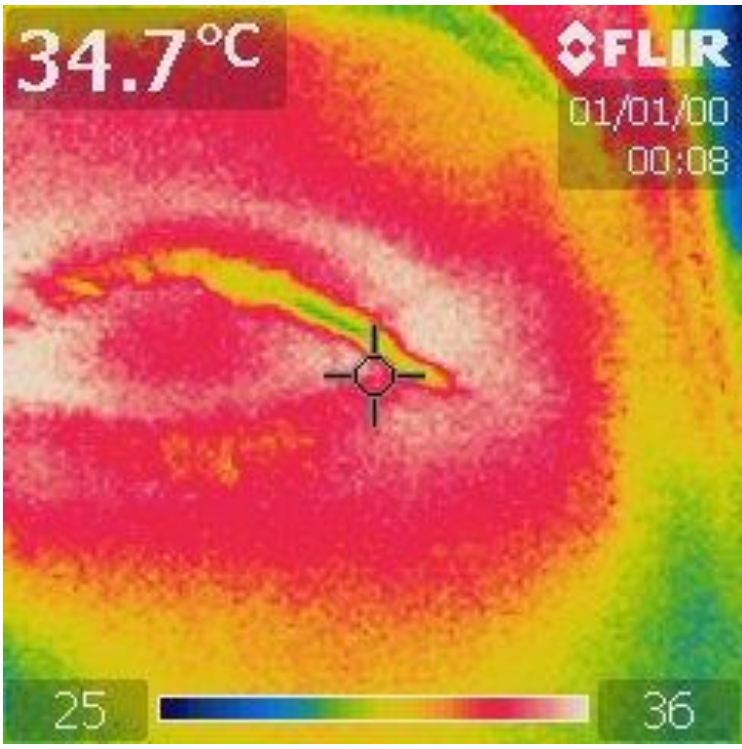

Figure 3: Temperature of the palpebral fissure

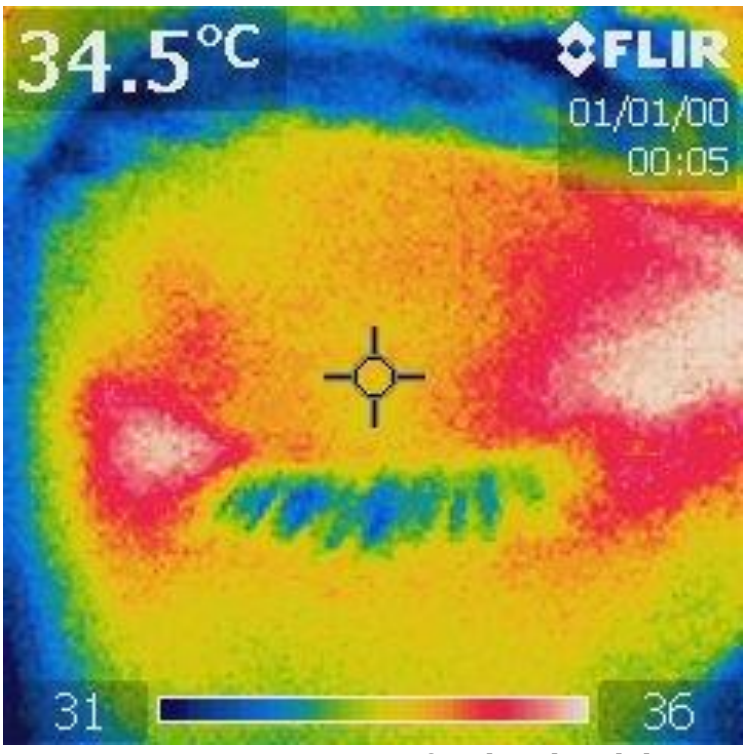

Figure 4: Temperature for closed eyelids

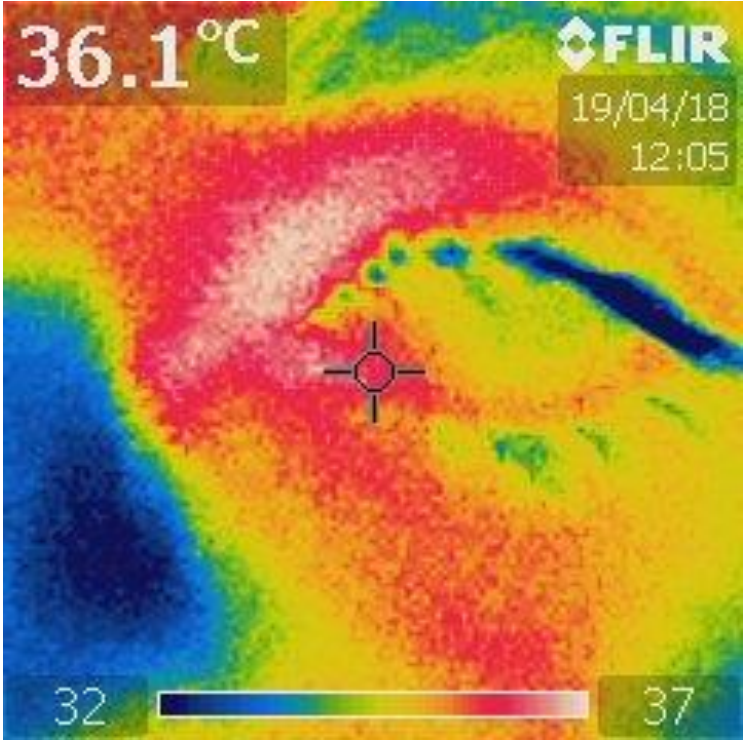

Figure 5: Caruncula lacrimalis temperature

\section{Graphical Results and Discussions}

First graphical results were achieved using FLIR camera software: Inspection Report for male subject temperature in the geometric center of the cornea, OD and OS, as it is shown in figure 6. Also, in figure 7 it is presented: Inspection Report for female subject temperature in the geometric center of the cornea, OD and OS. There were considered 10 male and 10 female subjects for this study with ages 20-25 years old.

Analysis of the evolution of temperature: the center of the cornea - the caruncula lacrimalis - the palpebral fissure are represented in figures 9 and 10 . The study of the temperature variation at the ocular surface level has generated the following conclusion: there is a peak value and proper with that of the body at the level of tear, its values being followed by those at the center of the cornea, and then by those at the level of the lateral commissure.

Finally, there are presented the temperature variation with time, figure 11 , considering blinking times : 1, 3,6 and 9 s. The accuracy of the recorded results is shown by the residue value (coefficient of determination) close to $1(\mathrm{R}=0.99184)$. This value was extracted from the statistical analysis of the regression equation $\mathrm{T}=-0.036735 \cdot \mathrm{t}+36.1245$, shown in figure 12 .

\section{Conclusions}

As a non-invasive method, IR-ray thermography can also be applied by the optometrist. The implementation of such a method of acquisition of thermographic images and, subsequently, the insertion of these images into a universal database would lead to a more efficient statistical analysis. Also, the Flir camera could also be used in the study of contactology, of the efficient adaptation of the patient with the different types of contact lenses.

The study of the variation of the temperature at the ocular surface level has led to the following conclusion: a peak value is recorded and close to that of the body at the level of the caruncula lacrimalis, these values being followed by those at the level of the cornea center, and then those at the level of the palpebral fissure.

These experimental results are shown in Figure 9 and Figure 10. The temperature difference between the caruncula lacrimalis and the palpebral fissure is strengthened by other studies, [5], [6] and is explained by a higher vascularisation in the nasal conjunctiva area. Also, much larger blood vessels from the nasal area, coming from two arteries, reinforce this explanation (the lateral part is oxygenated only by a single artery). Thus, in the area of the tear sack there is a higher temperature due to the higher evaporation rate and the increased instability of the tear film. 


\section{FF FistrRs}

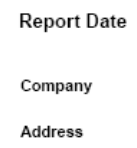

17.09 .2019

Address

Universitatea POLITEHNICA

Splaiul Independentei 313

Thermographer

Ciprian Ion Rizescu

Customer

Site Address

Contact Person

\section{Inspection Report}

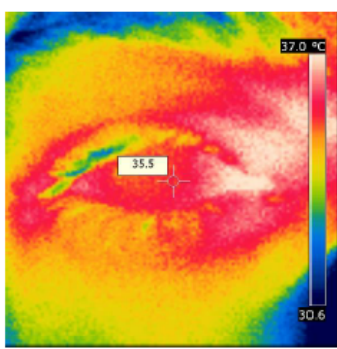

\begin{tabular}{|c|c|}
\hline Image and Object Parameters & Text Comments \\
\hline Camera Model & FLIR B-CAM Western, \\
\hline Image Date & 2000:01:01 00:05:23 \\
\hline Image Name & IR_0586.jpg \\
\hline Emissivity & 0,96 \\
\hline Reflected Temperature & $20,00^{\circ} \mathrm{C}$ \\
\hline Object Distance & $2,0 \mathrm{~m}$ \\
\hline
\end{tabular}

\section{Description \\ Right Eye M}

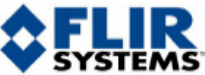

\section{Inspection Report}

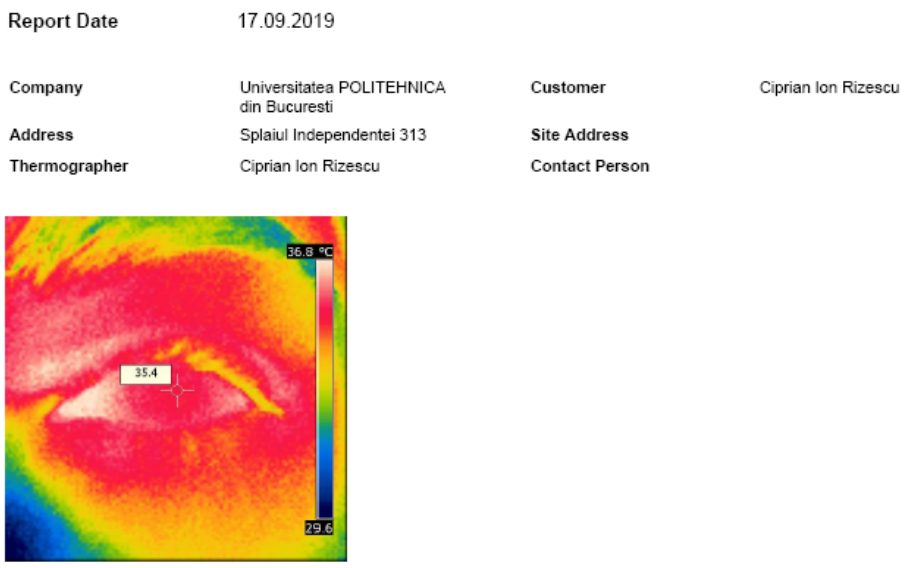

\begin{tabular}{|c|c|c|}
\hline \multicolumn{2}{|c|}{ Image and Object Parameters } & Text Comments \\
\hline Camera Model & FLIR B-CAM Western, & \\
\hline Image Date & 2000:01:01 00:05:31 & \\
\hline Image Name & IR_0587.jpg & \\
\hline Emissivity & 0,96 & \\
\hline Reflected Temperature & $20,0^{\circ} \mathrm{C}$ & \\
\hline Object Distance & $2,0 \mathrm{~m}$ & \\
\hline
\end{tabular}

\section{Description}

LEFT EYEM

Figure 6: Inspection Report for male subject temperature in the geometric center of the cornea, OD and OS 


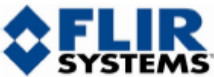

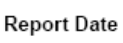

Company

Address

Thermographer

\section{Universitatea}

Splaiul Independentei 313

Ciprian Ion Rizescu

Customer

Site Address

Contact Person

\section{Inspection Report}

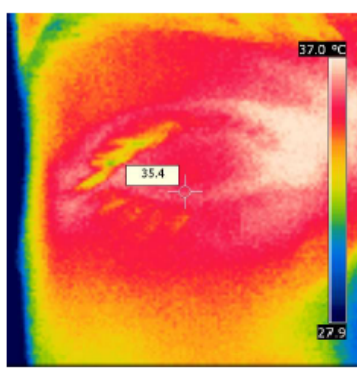

Image and Object Parameters

Camera Model FLIR B-CAM Western,

Image Date 2000:01:01 00:07:11

Image Name $\quad$ R__0590.jpg

Emissivity $\quad 0,96$

Reflected Temperature $\quad 20,0^{\circ} \mathrm{C}$

Object Distance $\quad 2,0 \mathrm{~m}$

\section{Description}

Right Eye F

\section{FF니ㄴㅛㅛ}

Inspection Report

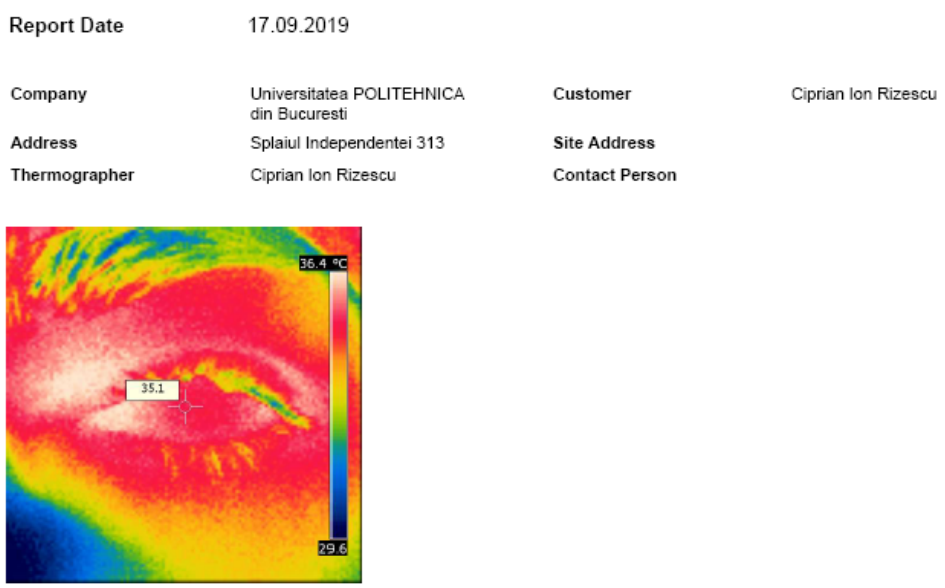

\begin{tabular}{|c|c|c|}
\hline Image and Object $\mathrm{P}$ & ameters & Text Comments \\
\hline Camera Model & FLIR B-CAM Western, & \\
\hline Image Date & 2000:01:01 00:07:25 & \\
\hline Image Name & IR_0592.jpg & \\
\hline Emissivity & 0,96 & \\
\hline Reflected Temperature & $20,0^{\circ} \mathrm{C}$ & \\
\hline Object Distance & $2,0 \mathrm{~m}$ & \\
\hline
\end{tabular}

Description

Left Eye $\mathrm{F}$

Figure 7: Inspection Report for female subject temperature in the geometric center of the cornea, OD and OS 


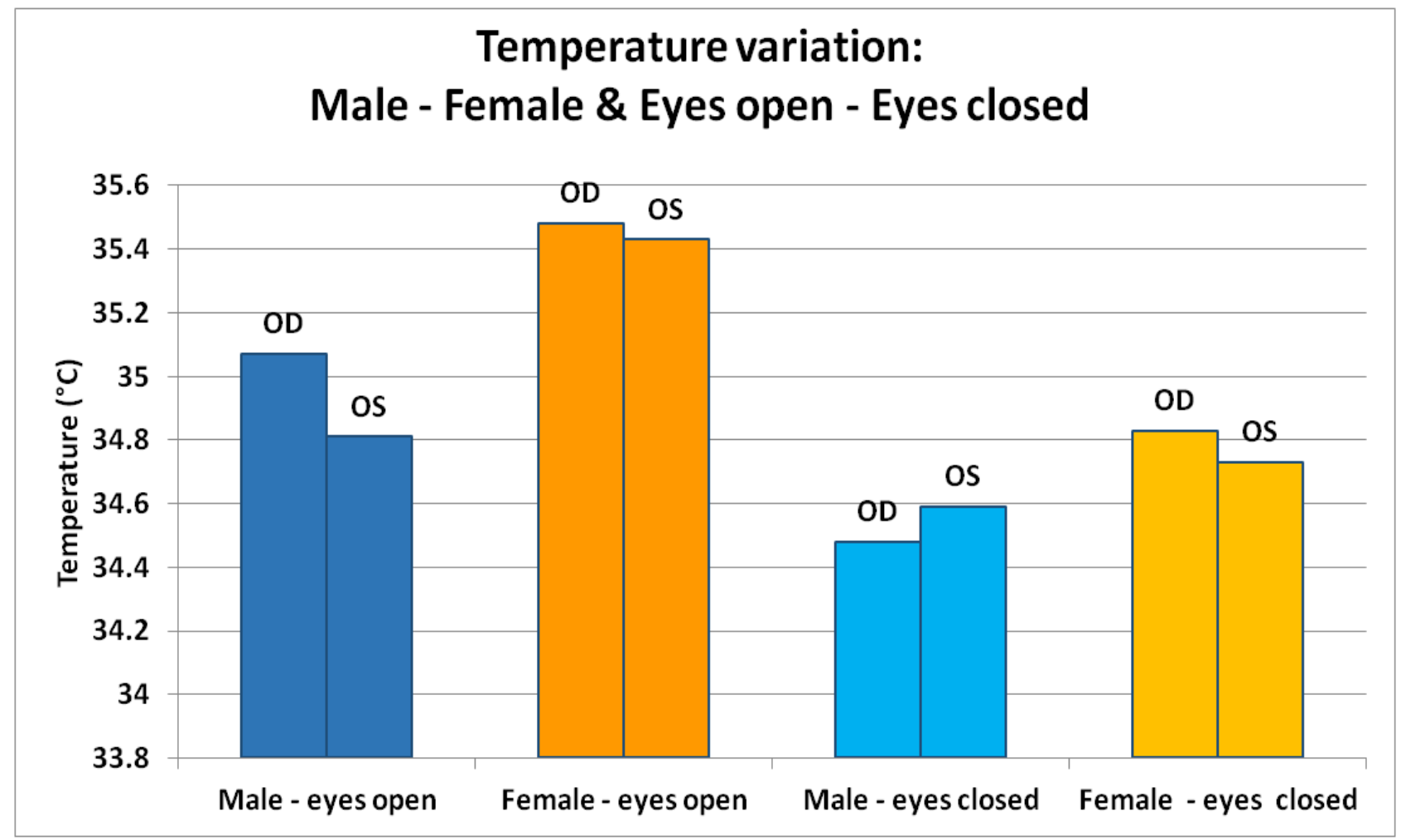

Figure 8: Temperature variation for male - female \& eyes open - eyes closed

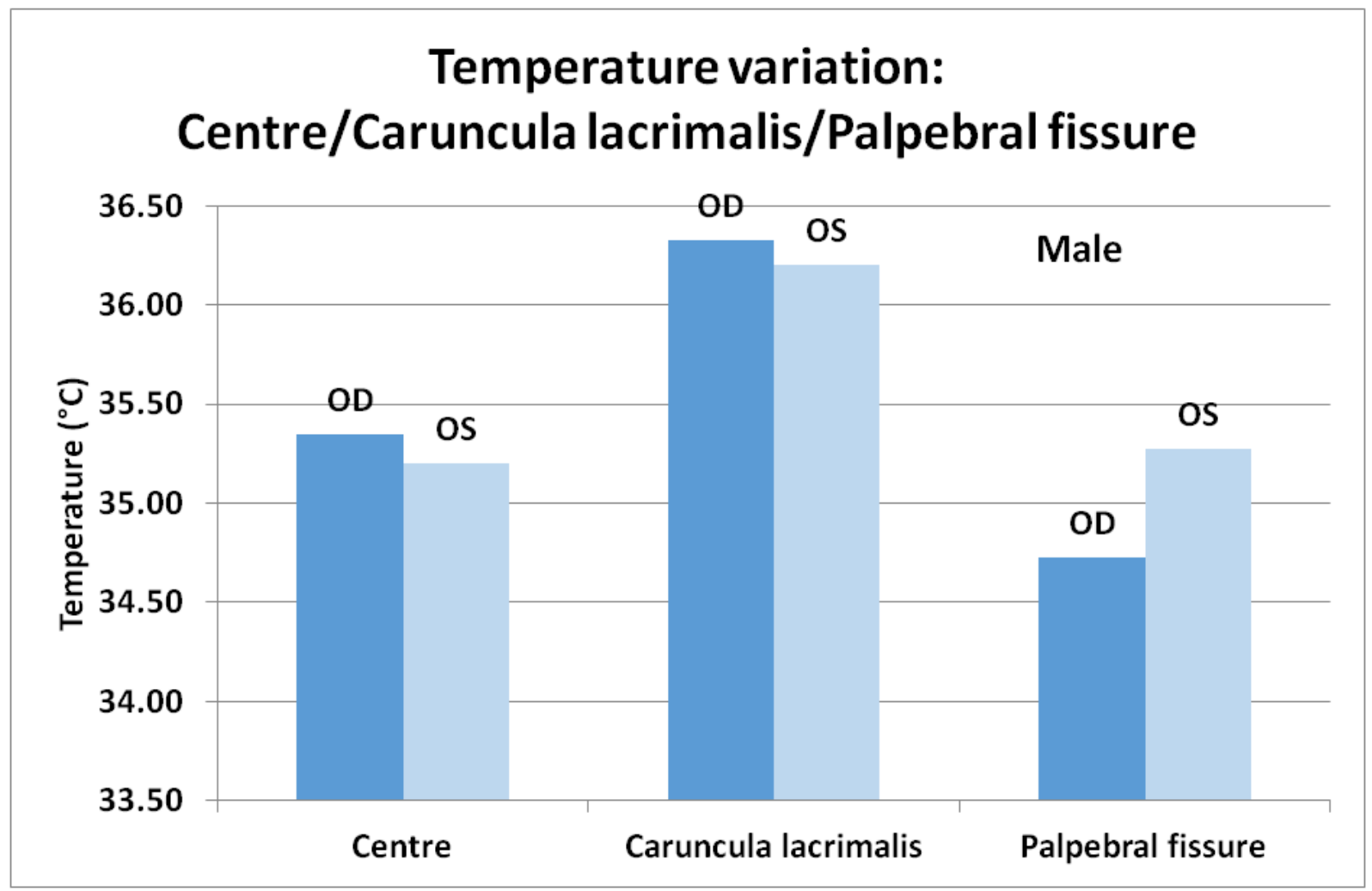

Figure 9: Temperature variation for male: centre / caruncula lacrimalis and palpebral fissure 


\section{Temperature variation: Centre/Caruncula lacrimalis/Palpebral fissure}

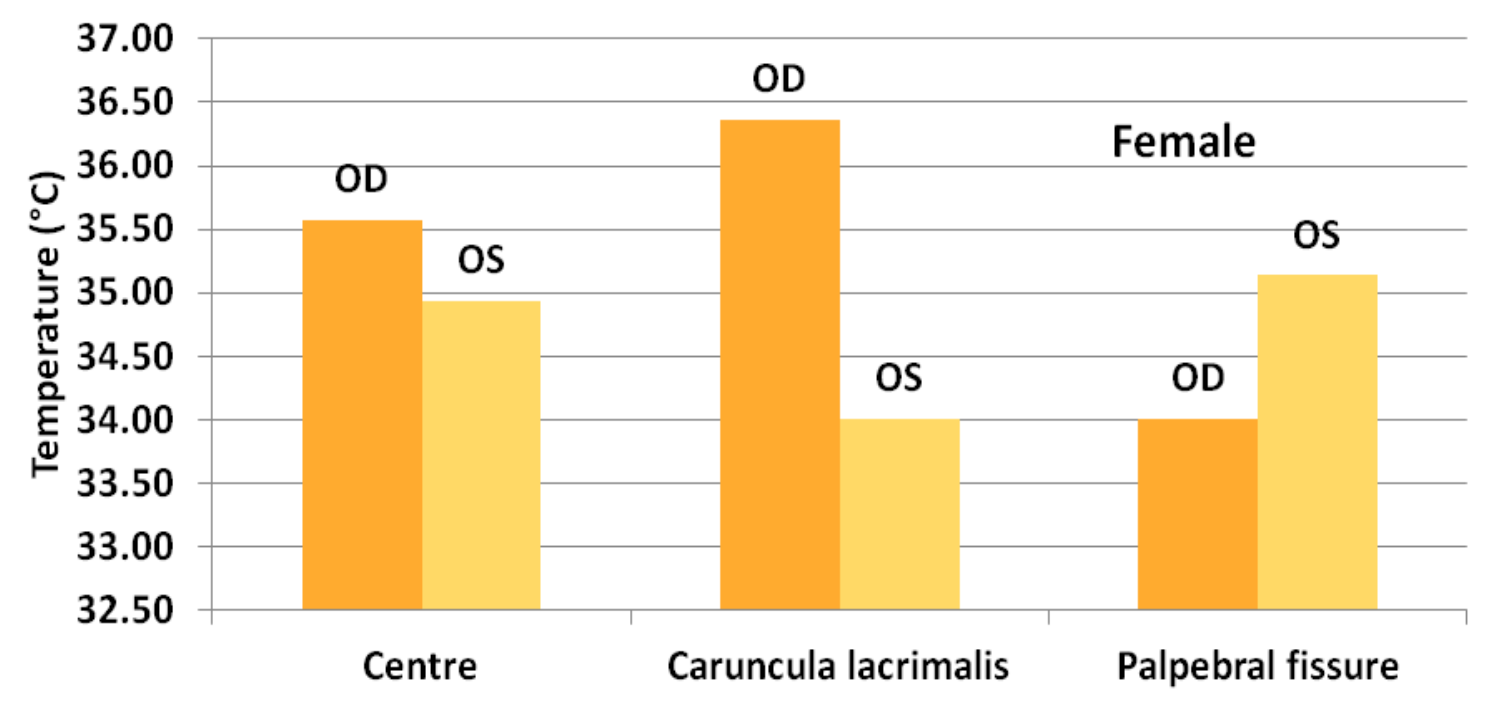

Figure 10: Temperature variation for female: centre / caruncula lacrimalis and palpebral fissure

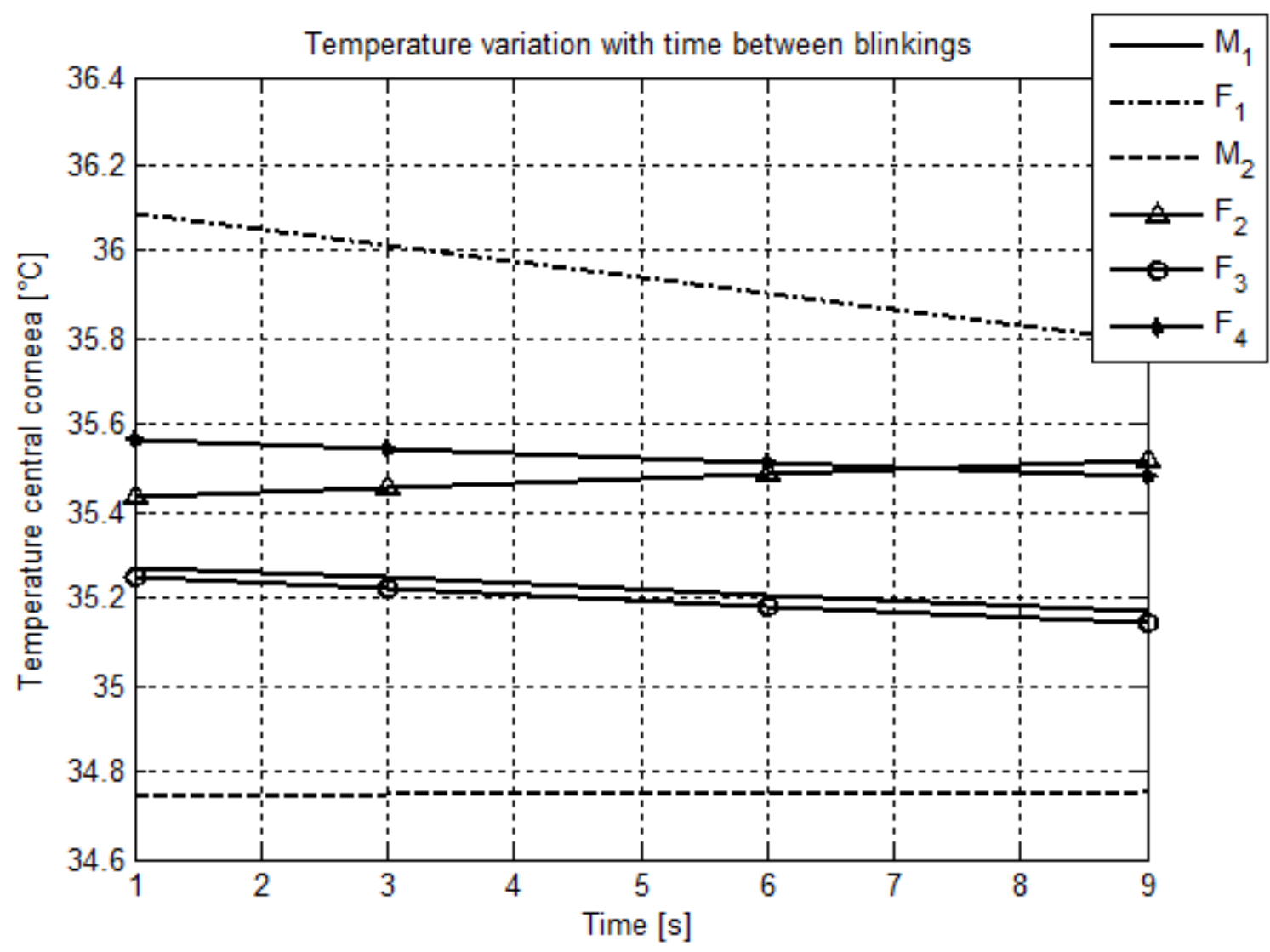

Figure 11: OST-time dependence between blinkings for 6 subjects, $(2 F, 4 M)$ 


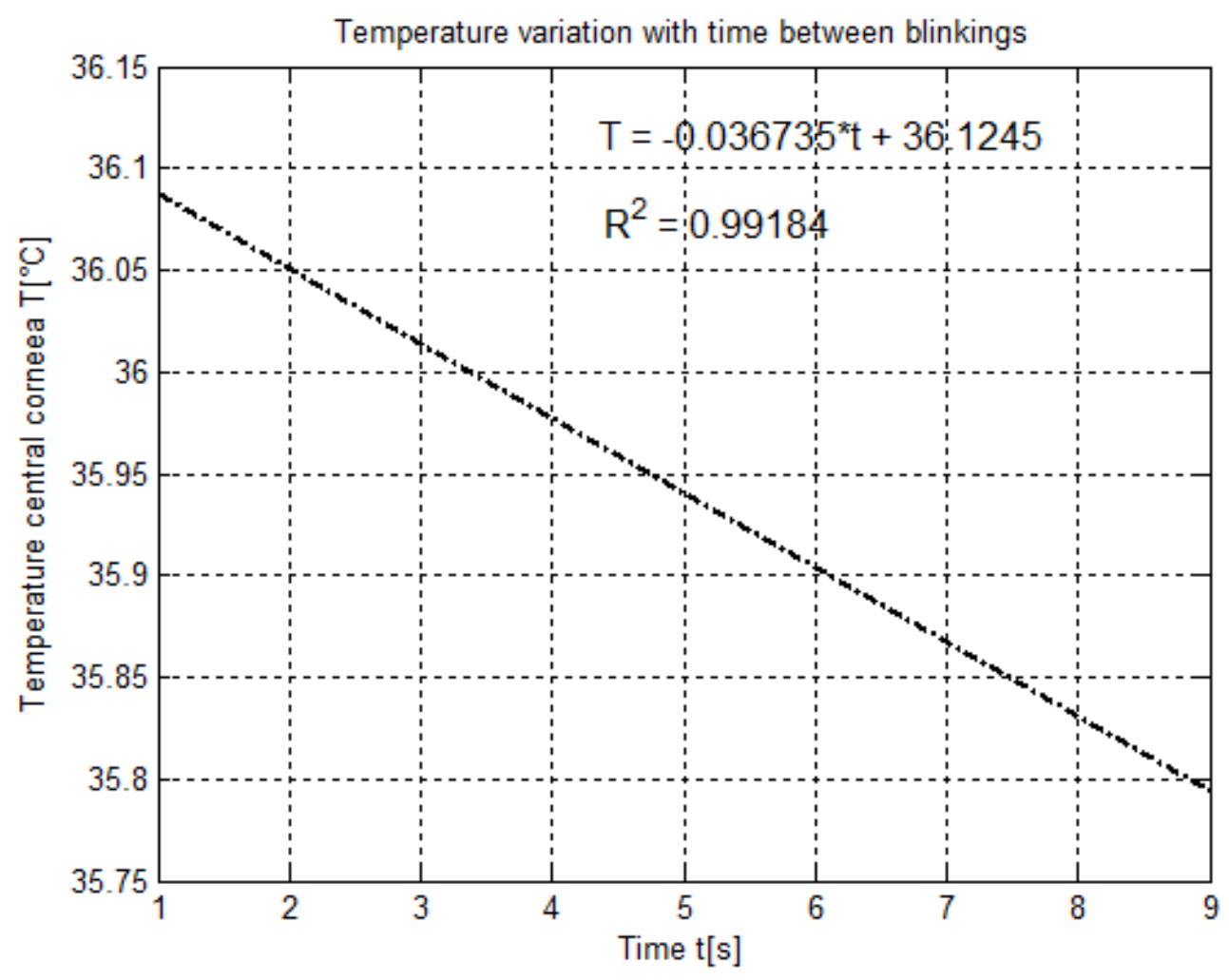

Figure 12: Linear regression equation for temperature evolution

Although the subjects considered in this study were exclusively healthy (ophthalmological), the further development of a 3D model and the analysis of the temperature gradient through a specialized platform would represent an evolutionary step in the analysis of the various states of ocular pathology.

An example of the pathology risk that will be studied is the long-term exposure to UV radiation, a simulation that will illustrate how the eye is thermally affected.

\section{References}

[1] Fernando Galassi, Barbara Giambene, Andrea Corvi, and Giacomo Falaschi, Evaluation of ocular surface temperature and retrobulbar haemodynamics by infrared thermography and colour Doppler imaging in patients with glaucoma, British Journal of Ophthalmology 2007; 91(7), pp: 878-881. doi: 10.1136/bjo.2007.114397
[2] Johanna Pattmöller, Jiong Wang, Elena Zemova, Berthold Seitz, Timo Eppig, Achim Langenbucher, Nóra Szentmáry, Correlation of corneal thickness, endothelial cell density and anterior chamber depth with ocular surface temperature in normal subjects, June 2014.

[3] Maria Jesus Giraldez, Shehzad A. Naroo, Carlos Garcia Resua, A preliminary investigation into the relationship between ocular surface temperature and lipid layer thickness, 2009

[4] Jen-Hong Tan, E.Y.K. Ng, U. Rajendra Acharya, C. Chee, Infrared thermography on ocular surface temperature: A review, Infrared thermography on ocular surface temperature: A review, December, 2008

[5] Li Li Tan, Srinivasan Sanjay, Philip B. Morgan, Screening for dry eye disease using infrared ocular thermography, February 2016

[6] Lili Tan, Zhi-Qiang Cai, Nai-Shin Lai, Accuracy and sensitivity of the dynamic ocular thermography and inter-subjects ocular surface temperature (OST) in Chinese young adults, 2009. 